\title{
Interplay between MPlase, YidC, and PMF during Sec-independent insertion of membrane proteins
}

\author{
Yuta Endo, Yuko Shimizu², Hanako Nishikawa², Katsuhiro Sawasato², Ken-ichi Nishiyama, (1)
}

\begin{abstract}
Integral membrane proteins with the $\mathrm{N}$-out topology are inserted into membranes usually in YidC- and PMF-dependent manners. The molecular basis of the various dependencies on insertion factors is not fully understood. A model protein, Pf3-Lep, is inserted independently of both YidC and PMF, whereas the V15D mutant requires both YidC and PMF in vivo. We analyzed the mechanisms that determine the insertion factor dependency in vitro. Glycolipid MPlase was required for insertion of both proteins because MPlase depletion caused a significant defect in insertion. On the other hand, YidC depletion and PMF dissipation had no effects on Pf3-Lep insertion, whereas V15D insertion was reduced. We reconstituted (proteo)liposomes containing MPIase, YidC, and/or $\mathrm{F}_{0} \mathrm{~F}_{1}$-ATPase. MPlase was essential for insertion of both proteins. YidC and PMF stimulated Pf3-Lep insertion as the synthesis level increased. V15D insertion was stimulated by both YidC and PMF irrespective of the synthesis level. These results indicate that charges in the $\mathrm{N}$-terminal region and the synthesis level are the determinants of YidC and PMF dependencies with the interplay between MPlase, YidC, and PMF.
\end{abstract}

DOI 10.26508/lsa.202101162 | Received 18 July 2021 | Revised 30 September 2021 | Accepted 1 October 2021 | Published online 12 October 2021

\section{Introduction}

Membrane proteins co-translationally are inserted into the cytoplasmic membranes of Escherichia coli with the aid of a series of insertion factors, such as signal recognition particle (SRP)/SRP receptor (SR), the SecYEG translocon and YidC (for reviews, see references 1,2 , and 3 ). In addition to these proteinaceous factors, glycolipid MPlase is involved in protein insertion $(4,5)$. As far as we know at present, MPlase cooperates with SecYEG and YidC to insert membrane proteins because MtIA insertion requires both MPlase and SecYEG $(6,7)$, and both MPlase and YidC are required for membrane insertion of the $c$ subunit of $F_{0} F_{1}$-ATPase $\left(F_{0} c\right)(8,9)$. Beside SecYEGdependent insertion, it is known that Sec-independent insertion occurs. In this case, it has been thought that membrane proteins are inserted into membranes spontaneously or unassisted through the hydrophobic interaction between membrane lipids and the transmembrane (TM) domains of proteins because a subset of membrane proteins, such as $\mathrm{M} 13$ procoat, $\mathrm{Pf3}$ coat and $\mathrm{F}_{0} \mathrm{C}$, are inserted into liposomes of phospholipids only $(10,11,12)$. However, inclusion of DAG in liposomes at a physiological level blocks spontaneous insertion completely $(6,13,14)$, indicating that spontaneous insertion does not occur in vivo. Alternatively, MPlase $(15,16)$ and YidC $(17,18)$ are involved in the insertion. We have observed a cooperative function of MPlase with YidC, in which MPlase functions at an early stage and then the substrate proteins are transferred to YidC to complete insertion $(7,8,9)$.

Sec-independent insertion is still not fully understood. M13 procoat and Pf3 coat are inserted into membranes in YidC- and proton motive force (PMF)-dependent manners $(19,20)$, whereas the respective mutants, such as $3 \mathrm{~L}-\mathrm{Pf} 3$ coat, sometimes render insertion YidC-independent or PMF-independent or even independent of both $(21,22)$. $F_{0} C$ insertion depends upon YidC but not PMF (23). Therefore, the mechanisms underlying these differences in dependencies on YidC and PMF are totally unknown. Although we have shown that MPlase is involved in the insertion of abovementioned proteins $(6,7,8,16)$, it remains unknown whether or not MPlase is generally involved in all the types of insertion described above, that is, it is unknown whether YidC/Sec-independent, YidC only, YidC/PMF only, or YidC/Sec mechanisms co-exist and operate in an MPlase-dependent manner. The insertion factor dependencies for proteins described above were summarized in Table 1. MPlase was essential for insertion all of them, whereas the dependencies on SecYEG, YidC, and PMF quite differ.

A model substrate protein, Pf3-Lep, is composed of the periplasmic region of Pf3 coat protein, followed by TM1 of Lep (leader peptidase) (24). Whereas Pf3 coat insertion is YidC-dependent (17, 22), Pf3-Lep insertion is not affected by YidC depletion (24). In the case of PMF, Pf3 coat insertion is significantly stimulated by PMF, but Pf3-Lep insertion is not affected by PMF dissipation (14, 24). On the other hand, the V15D mutant of Pf3-Lep is severely affected by both YidC depletion and PMF dissipation (24). These differences in insertion factor dependencies were also summarized in Table 1. Therefore, these model proteins are good substrates to analyze the switching of insertion dependency. In this study, we analyzed the molecular mechanisms underlying the membrane insertion of

${ }^{1}$ The United Graduate School of Agricultural Sciences, Iwate University, Morioka, Japan ${ }^{2}$ Department of Biological Chemistry and Food Science, Faculty of Agriculture, Iwate University, Morioka, Japan

Correspondence: nishiyam@iwate-u.ac.jp 
Table 1. Insertion factor dependency for membrane proteins determined in the in vitro reconstitution system.

\begin{tabular}{llllll} 
Protein & SecYEG & MPlase & YidC & Proton motive force & References \\
\hline MtlA & Essential & Essential & Conditional & No effect & 6 and 7 \\
\hline Pf3 coat & Not essential & Essential & Stimulate & Stimulate & 7 and 22 \\
\hline 3L-Pf3 coat & Not essential & Essential & Conditional & No effect & $7,15,16$, and 22 \\
\hline M13 procoat & Not essential & Essential & Stimulate & Stimulate & 6,7, and 14 \\
\hline Fo & Not essential & Essential & Stimulate & No effect & 8, and 23 \\
\hline Pf3-Lep & Not essential & (This study) & Not required & No effect & 24 \\
\hline Pf3-Lep V15D & Not essential & (This study) & Stimulate & Stimulate & 24 \\
\hline
\end{tabular}

The insertion factor dependencies for specified membrane proteins are indicated. "Conditional" denotes that YidC stimulates insertion when the substrate level is high. In the case of Pf3-Lep and Pf3-Lep V15D, used in this study, the in vivo results are indicated.

these substrates to clarify the switching, by means of an in vitro system. We found that both substrates are inserted into membranes in an MPlase-dependent manner, and that charges in the $\mathrm{N}$-terminal region and the synthesis level are determinants of the YidC and PMF dependencies with the interplay between MPlase, YidC, and PMF.

\section{Results}

\section{In vitro analysis of Pf3-Lep (amber) and V15D insertion}

Pf3-Lep (amber) is a model membrane protein, in which the periplasmic region of Pf3 coat protein is followed by the first TM and the cytoplasmic region of Lep with three stop codons after 79E (Fig $1 \mathrm{~A}$, left; Fig S1). An amino acid substitution of $15 \mathrm{~V}$ with $\mathrm{D}$ was introduced into the V15D mutant (Fig 1A, right). To examine the dependency on insertion factors, MPlase-depleted (KS23) and YidCdepleted (JS7131) INV (inverted and inner membrane vesicles) were prepared (Fig 1B). The successful depletion of either MPlase or YidC was confirmed by immunoblotting (Fig 1B). Up-regulation of MPlase was also observed upon YidC depletion, as reported (7). The insertion activity was determined by means of a protease protection assay (Fig 1C) (25). The membrane insertion region is protected by proteinase K (PK), giving membrane-protected fragments (MPFs). At first, insertion of both proteins into the wild-type INV (EK413) was examined. Upon PK digestion, mainly three bands appeared (Fig 1D). Band " $\mathrm{C}$ " appeared only in the presence of INV, indicating that this band represents the MPF. Band " $\mathrm{i}$ " was a membrane-embedded fragment digested at the $N$ terminus. Because the intensity of this band was very weak, we did not characterize this material further. Band " $r$ " appeared even in the absence of INV, indicating that this band does not correspond to any insertion, but that it is a PK-resistant material derived from the hydrophobic TM domain. This band is indicated by an asterisk at the left of each gel. Hereafter, we focused on the appearance of the " $c$ " band as an index of membrane insertion. When SRP/SR (Ffh/FtsY) was added to the reaction mixture, a significant increase in the insertion of both proteins was observed, indicating that the TM domain was recognized by SRP to solubilize substrates. Therefore, we added SRP/ $\mathrm{SR}$ in the following experiments. When YidC-depleted INV were used, no inhibition of Pf3-Lep (amber) insertion was observed, whereas a decrease in V15D insertion was observed (Fig 1E), consistent with in vivo results (24). On the other hand, when MPlasedepleted INV were used, a significant decrease in insertion of both substrates was observed, strongly suggesting that these substrate proteins require MPlase for insertion (Fig 1E).

Next, we examined whether SecA and PMF are involved in Pf3Lep (amber) insertion (Fig 2). SecA activity is inhibited by sodium azide (26). When azide was added to the reaction mixture, pOmpA translocation was completely abolished (Fig 2A). Under our experimental conditions, PMF is generated by $\mathrm{F}_{0} \mathrm{~F}_{1}$-ATPase, of which the activity is abolished by $\mathrm{N}, \mathrm{N}$-dicyclohexylcarbodiimide (DCCD) (27). Insertion of Pf3 coat protein is stimulated by the membrane potential, a component of PMF $(10,20)$. When DCCD was added to the reaction mixture, $\mathrm{Pf} 3$ coat insertion was significantly reduced (Fig 2B). Insertion of both Pf3-Lep (amber) and V15D was not affected by the addition of azide, indicating that SecA is not involved in the insertion of these proteins (Fig 2C). DCCD addition did not affect Pf3-Lep (amber) insertion, indicating that Pf3-Lep (amber) insertion is PMF-independent (Fig 2D, left). On the other hand, V15D insertion was reduced by $\sim 2 / 3$, indicating that $P M F$ is involved in V15D insertion (Fig 2D, right). When $\triangle$ YidC INV were used, Pf3-Lep (amber) insertion was even affected by DCCD addition, indicating that efficient insertion of Pf3-Lep (amber) requires PMF in the absence of YidC (Fig 2E, left). In the case of V15D insertion, the insertion into $\triangle$ YidC INV was as low as that into WT INV in the absence of PMF, indicating that both YidC and PMF are involved in V15D insertion. In summary, Pf3-Lep (amber) insertion is independent of both YidC and PMF, but is reduced in the absence of both. On the other hand, V15D insertion requires both YidC and PMF for the efficient insertion in the in vitro assay system using INV. These results also suggest that the YidC function is tightly linked with PMF utilization for stimulation of membrane insertion.

\section{Reconstitution analysis reveals interplay between MPIase, YidC and PMF}

Next, we tried to reconstitute Pf3-Lep (amber) and V15D insertion (Fig 3). When liposomes composed of only phospholipids (PL) were used, efficient spontaneous insertion of both proteins, which does not reflect the in vivo reaction $(6,13)$, was observed (Fig 3A, "PL"). This disordered spontaneous insertion was blocked by the presence of DAG ("PL+DAG"). When YidC was included in $P L+D A G$ 


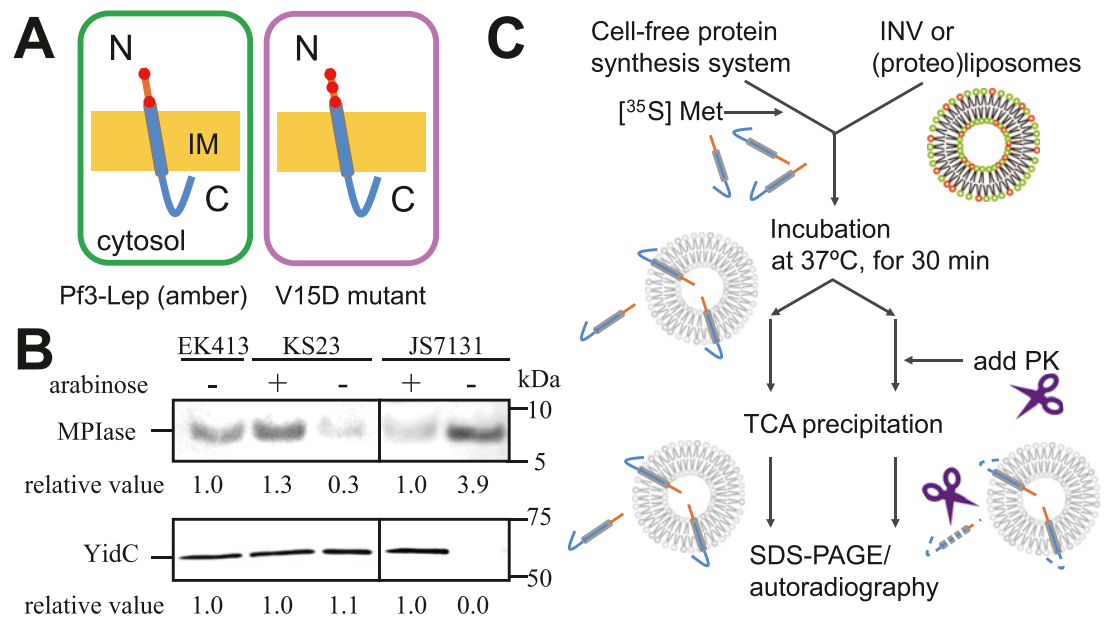

Figure 1. MPlase is involved in membrane insertion of
Pf3-Lep (amber)

(A) Membrane topologies of Pf3-Lep (amber) (left) and its V15D mutant (right). The $\mathrm{N}$-terminal region is exposed to the periplasm and the $\mathrm{C}$-terminal region to the cytosol. Negatively charged residues in the periplasmic region are denoted by red dots. (B) Preparation of MPlase- and YidC-depleted INV. Depletion of MPlase (upper panel) and YidC (lower panel) was confirmed by immunoblotting. Note that MPlase is up-regulated by YidC depletion. The relative levels of MPlase and YidC are shown below the blots. Whole cell extract $(1 \mu \mathrm{g}$ protein) and INV (10 $\mu \mathrm{g}$ protein) were used to detect MPlase and YidC, respectively. (C) Schematic representation of assaying of membrane insertion in vitro. A membrane-protected fragment (MPF) arises upon PK digestion. The C-terminal region of Pf3-Lep (amber) is digested, giving the MPF. (D) Membrane insertion of both Pf3-Lep (amber) and V15D is stimulated by signal recognition particle. The substrates were in vitro synthesized in the presence of INV prepared from EK413 (WT) as specified. Upon PK digestion after synthesis, three bands, "i," "c," and "r," appeared. Band "i" represents incomplete insertion, while band " $\mathrm{C}$ " represents the MPF. Band " $r$ " is the PK-resistant band because this non-specifically appeared even in the absence of membranes. Hereafter, this band is indicated by asterisks. The insertion activity (the percentage of the level of " $c$ " as to that of the substrates) is shown below the autoradiograms. The numbers of methionine (three in the substrates and two in MPF) were considered in the calculation. The positions of molecular weight markers are also shown

E

\begin{tabular}{|c|c|c|c|c|c|c|c|c|c|}
\hline Substrate & \multicolumn{4}{|c|}{ Pf3-Lep (amber) } & \multicolumn{4}{|c|}{ V15D } & \\
\hline SRP/SR & \multicolumn{2}{|c|}{+} & \multicolumn{2}{|c|}{ - } & \multicolumn{2}{|c|}{+} & \multicolumn{2}{|c|}{-} & \\
\hline INV & WT & - & WT & - & WT & - & WT & - & \\
\hline $\mathrm{PK}$ & \begin{tabular}{l|l}
- & + \\
\end{tabular} & \begin{tabular}{l|l|}
- & + \\
\end{tabular} & \begin{tabular}{l|l}
- & + \\
\end{tabular} & + & \begin{tabular}{l|l|}
- & + \\
\end{tabular} & \begin{tabular}{l|l}
- & + \\
\end{tabular} & \begin{tabular}{l|l|}
- & + \\
\end{tabular} & + & \\
\hline $\begin{array}{l}\text { Pf3-Lep } \\
\text { (amber)/- } \\
\text { V15D }\end{array}$ & $\infty$ & 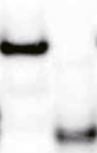 & 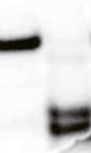 & 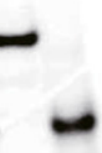 & * & $=$ & - & tise & $=$ \\
\hline inse & 25.4 & 0.6 & 10.6 & 0.5 & 19.3 & 0.8 & 10.8 & 0.4 & \\
\hline
\end{tabular}

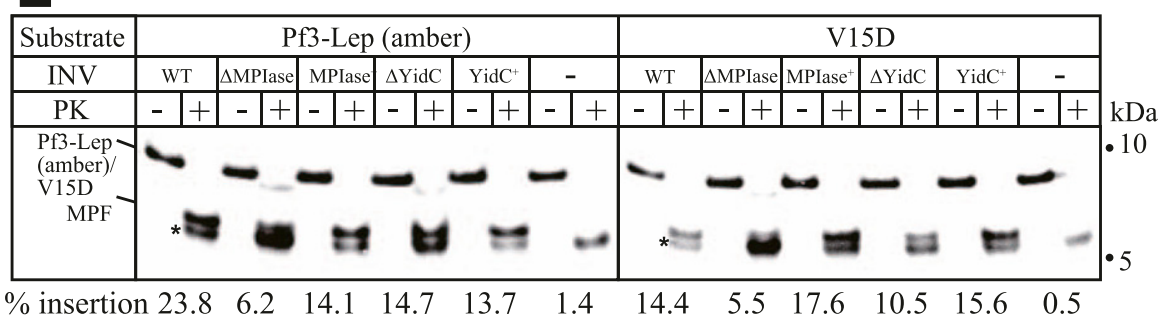

by dots. (E) Membrane insertion of Pf3-Lep (amber) and V15D into MPlase- and YidC-depleted INV. (B) The substrates were in vitro synthesized in the presence of $\triangle M$ Plase INV and $\triangle$ YidC INV, shown in (B), as specified. (D) The insertion activity was determined as described in (D) and is shown.

Source data are available for this figure. liposomes, no insertion activity for either protein was observed, indicating that YidC is not sufficient for insertion (Fig 3B, left). On the other hand, both proteins were inserted into MPlase liposomes, albeit at a low level (basal level) (Fig 3B, middle), indicating that MPlase is essential for the insertion of both proteins. These activities reached a plateau level because an increase in the amount of MPlase had no effect on the activity (Fig 3B, right). When YidC was co-reconstituted with MPlase (+YidC), Pf3-Lep (amber) insertion was stimulated (Fig 3B, middle and right) to a level comparable with that into WT INV (Figs 1 and 2). On the other hand, V15D insertion was not significantly stimulated by YidC (Fig 3B, middle and right), unlike the case of WT INV (Figs 1 and 2). We assumed that YidC had little effect on V15D insertion because of the absence of PMF. We imposed PMF by co-reconstituting $\mathrm{F}_{0} \mathrm{~F}_{1}$-ATPase together with MPlase and/or YidC (Fig 3C). When Pf3-Lep (amber) insertion was analyzed, the maximum activity was obtained in the presence of MPlase only, similarly to in the presence of both MPlase and YidC (Fig 3C, left). Although the activity of V15D insertion was as low as that in the absence of YidC, it was clearly stimulated to the level into WT INV in the presence of both MPlase and YidC (Fig 3C, right). Thus, the results in the reconstitution experiments reproduced those using
INV, that is, we found that a sufficient level of Pf3-Lep (amber) insertion is obtained when either MPlase and YidC or MPlase and PMF are present. On the other hand, all three factors, MPlase, YidC, and PMF, are necessary for a sufficient level of V15D insertion.

We also found that the expression level of the substrate proteins is an important parameter that determines the insertion efficiency (Fig 4). When the expression level was increased by increasing the amounts of radioactive methionine $(\sim 2 \mathrm{MBq} / \mathrm{ml}$ in Figs $1-3$, and $\sim 10$ $\mathrm{MBq} / \mathrm{ml}$ in Fig 4), the expression level increased from 0.34 0.71 $\mathrm{pmol} / \mathrm{ml}$ (Figs 1-3) to 4.1 4.9 $\mathrm{pmol} / \mathrm{ml}$ (Fig 4A). In this case, Pf3-Lep (amber) insertion into MPlase/YidC proteoliposomes in the absence of PMF was as efficient as in the presence of PMF, however, insertion into MPlase liposomes remained low even in the presence of PMF (upper panel), unlike as shown in Fig 3C, indicating that an increase in the substrate amounts rendered Pf3-Lep (amber) insertion YidC-dependent. The maximum activity of V15D insertion was obtained only when the three factors, MPlase, YidC and PMF, were present (lower panel). Whereas V15D insertion was observed when MPlase was present, YidC addition only or PMF imposition only had little effect on V15D insertion. When cold methionine was added to the reaction mixture, the synthesis level was further 


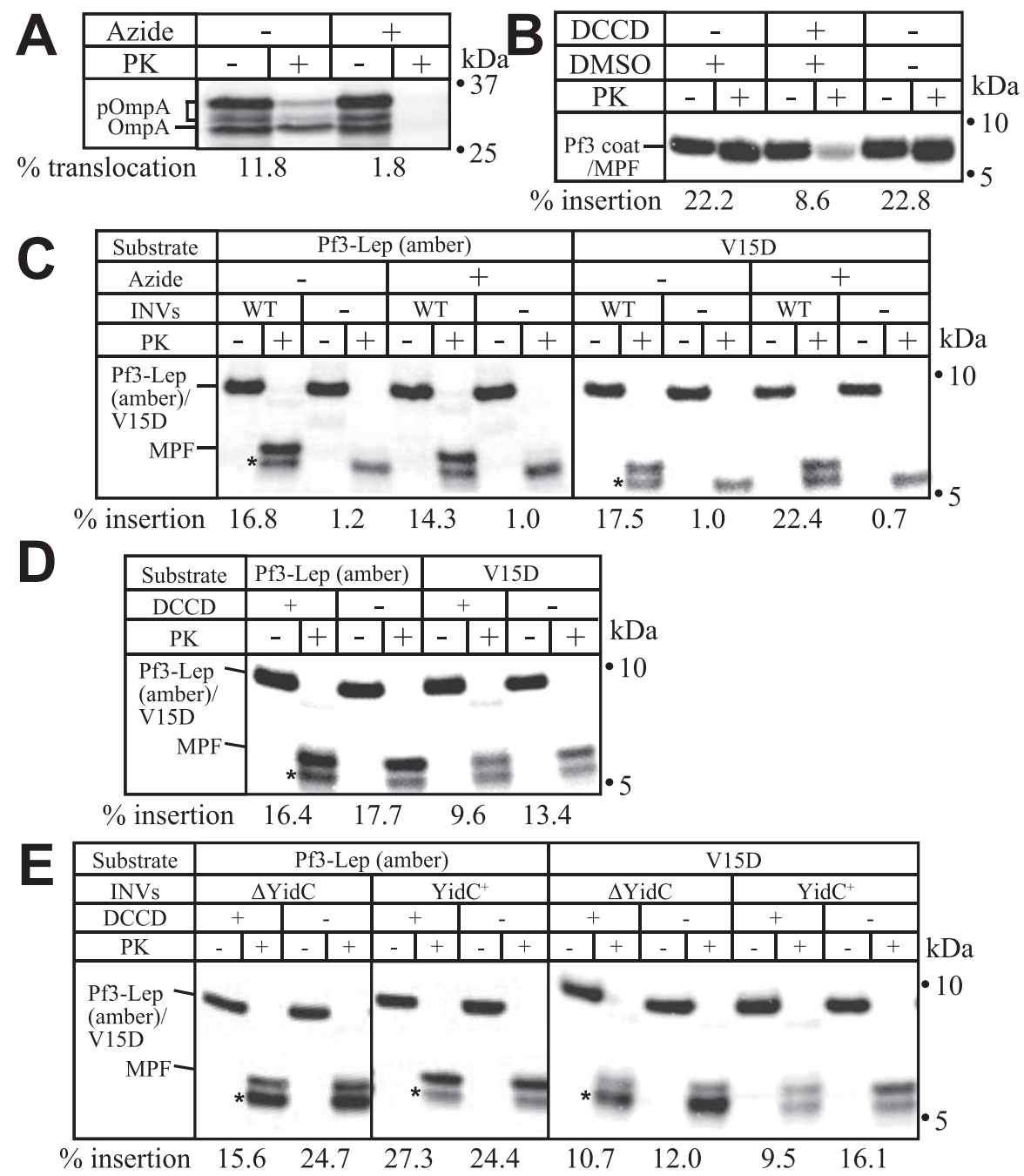

Figure 2. Effects of SecA and proton motive force (PMF) on Pf3-Lep (amber) and V15D insertion.

(A) Effect of sodium azide on SecA-dependent pOmpA translocation. pOmpA was in vitro synthesized in the presence of INV prepared from EK413, followed by the PK protection assay. Sodium azide ( $1 \mathrm{mM})$ was added as specified. The percentage of the translocated materials (pOmpA plus OmpA) is shown at the bottom. The numbers of methionine (six in pOmpA and five in OmpA) were considered in the calculation. (B) Effect of DCCD on PMF-dependent stimulation of Pf3 coat insertion. Pf3 coat was in vitro synthesized in the presence of INV prepared from EK413, followed by the PK protection assay. DCCD $(0.15 \mathrm{mM})$ or DMSO was added as specified. The insertion activity was determined and is shown at the bottom. (C) Effect of sodium azide on Pf3-Lep (amber)/V15D insertion. The insertion activity for Pf3-Lep (amber) (left half) and V15D (right half) was determined as described in the legend to Fig $1 \mathrm{C}$, and is shown at the bottom. (A) Where specified, sodium azide was added as in (A). The position of membrane-protected fragment is indicated. The PKresistant bands that appeared in the absence of INV as indicated by asterisks. (D, E) Effect of DCCD on Pf3Lep (amber)/V15D insertion. The insertion activity for Pf3-Lep (amber) (left half) and V15D (right half) was determined as described in the legend to Fig $1 D$, and is shown at the bottom. (B) DCCD was added as in (B). (D, E) INV prepared from EK413 were used in (D), whereas $\triangle$ YidC or YidC ${ }^{+}$INV prepared from JS7131 were used in (E) as specified. The position of membraneprotected fragment is indicated. The PK-resistant bands unrelated with membrane insertion are indicated by asterisks. increased to 390 530 pmol/ml (Fig 4B). In this case, the presence of both MPlase and YidC did not lead to the maximum activity of Pf3Lep (amber) insertion found in the presence of the three factors (upper panel), unlike as shown in Fig 4A. Similarly, V15D insertion required the three factors (lower panel). These results indicate that the MPlase-dependent insertion of both substrate proteins becomes dependent on both YidC and PMF as the expression level of the substrates increased.

\section{MPlase and YidC interact directly}

The results so far obtained reveal the presence of functional interaction between MPIase and YidC. Therefore, we examined whether or not MPlase and YidC directly interact by means of the pull-down assay. JS7131, a yidC-disrupted strain, expresses YidC at the wild-type level in the presence of arabinose (17). When plasmid pTac-YidC-CHis was used to transform in JS7131, the transformants grew very well in the absence of inducer IPTG with leaky expression of His-tagged YidC at the wild-type level
(Fig 5A). INV were prepared from both strains, followed by the pull-down assay. When solubilized membranes were subjected to cobalt metal-affinity column chromatography, His-tagged YidC was purified from sample of JS7131/pTac-YidC-CHis, but not from that of JS7131, as expected (Fig 5B). The same fraction was also analyzed to detect MPlase, by immunostaining of the TLC plates. Fig $5 \mathrm{C}$ clearly shows that the fraction with His-tagged YidC contains MPlase, indicating that YidC and MPlase were coprecipitated. These results strongly suggest that MPlase and YidC interact directly in the membranes.

\section{Discussion}

In this study, we examined the molecular mechanism underlying the insertion of $\mathrm{N}$-out membrane proteins using Pf3-Lep (amber) and its mutant V15D as substrates by means of INV and reconstituted (proteo)liposomes. All the results indicated that glycolipid MPlase is essential for the insertion of both proteins. Under 


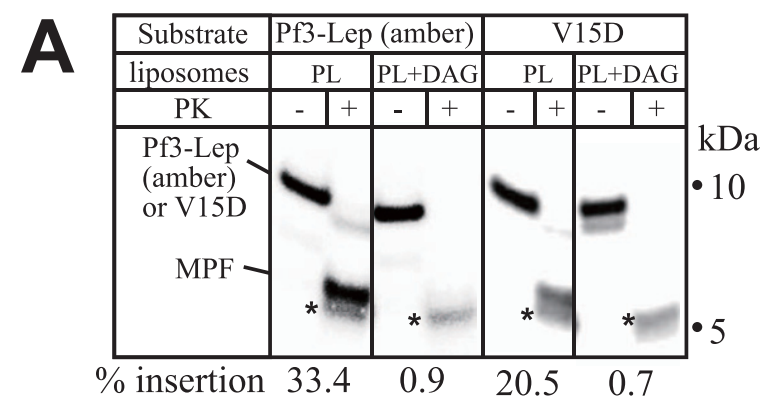

B
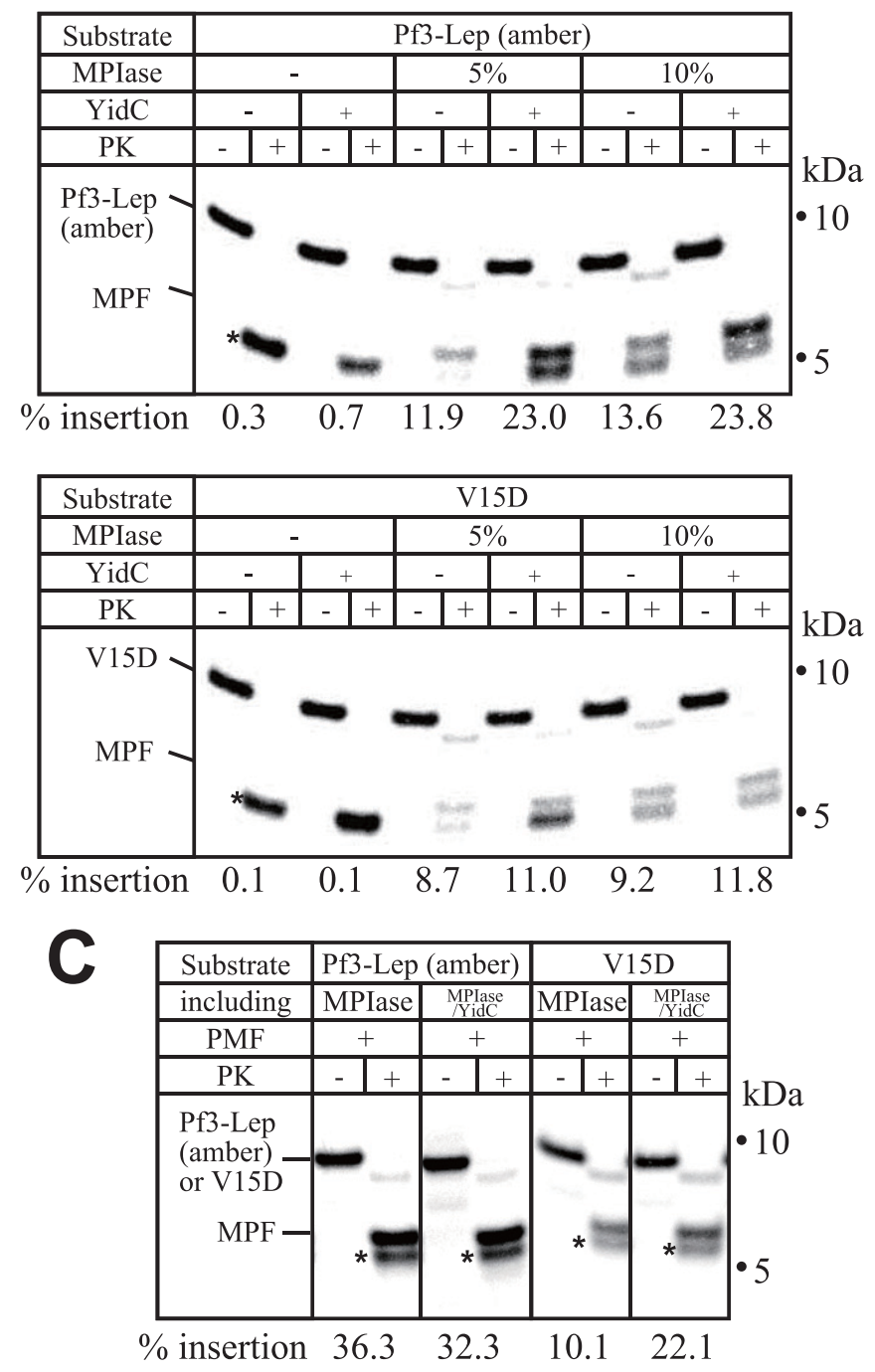

Figure 3. Reconstitution of Pf3-Lep (amber) and V15D insertion.

(A) Spontaneous insertion of both Pf3-Lep (amber) and V15D is blocked by a physiological level of DAG. Pf3-Lep (amber) (left half) and V15D (right half) were in vitro synthesized in the presence of liposomes formed with phospholipids ( $\mathrm{PL}$ ) or phospholipids and DAG (PL+DAG), followed by the PK protection assay. The insertion activity was determined and is shown at the bottom. The position of membrane-protected fragment is indicated. The PK-resistant bands unrelated with membrane insertion are indicated by asterisks. (B) Effects of MPlase and YidC on Pf3-Lep (amber) and V15D insertion. (Proteo)liposomes containing MPlase and YidC were reconstituted, followed by assaying of Pf3-Lep (amber) (upper panel) and V15D (lower panel) insertion. The insertion activity was determined as described in the legend to Fig 1D and is shown at the bottom. all the conditions examined, both Pf3-Lep (amber) and the V15D mutant were inserted into MPIase liposomes, albeit to a basal level. To achieve efficient insertion, YidC and PMF are required in addition. The importance of YidC and PMF was determined by the presence of the charge at the $\mathrm{N}$-terminal domain and the expression level. The presence of the charge (V15D in this case) and the higher expression level of the substrates render the insertion more dependent on YidC and PMF. Thus, the interplay of the three factors, MPIase, YidC, and PMF, causes efficient insertion.

Interplay between MPlase and YidC is supported by the fact that YidC depletion causes MPlase upregulation (7), suggesting the occurrence of functional interaction between MPlase and YidC. Pull-down assay actually supported the direct interaction between MPlase and YidC. The early stage of insertion is initiated by MPlase, followed by the YidC function to complete insertion at the late stage $(7,8)$. Because PMF was not imposed in these studies, interaction of substrate proteins with MPlase and YidC occur even in the absence of PMF, as seen in this study as well. The YidC function is powered by PMF, as indicated by the crystal structure of YidC $(18,28)$. The positive charge in the cavity in the membrane-embedded domain of YidC interacts with the negative charge found in the $\mathrm{N}$-terminal region of the membrane protein through an electrostatic interaction. Then, the negative charge is translocated to the periplasmic space to complete insertion. This step would be accelerated with the aid of PMF (18). This model also explains that PMF stimulates insertion more effectively in the presence of YidC. As a result, MPlase and YidC could function for the next insertion reaction. This model implies that YidC and PMF become less important for insertion when the expression level of the substrates is lower, or the number of negative charges in the $\mathrm{N}$-terminal region decreases (Fig 6). In an extreme case, only MPlase is even sufficient for insertion (Fig 6, left box). The insertion of some membrane proteins, such as 3L-Pf3 coat (10), is independent of both YidC and PMF (22), similar to in the case for Pf3-Lep (24), suggesting that the YidCindependent mechanism is operative. In this case, MPlase should be required as shown in this study. On the other hand, we have shown that $3 \mathrm{~L}-\mathrm{Pf} 3$ coat insertion becomes YidC-dependent when the expression level increases (7). Thus, interplay between MPlase, YidC, and PMF is important for the membrane insertion of $\mathrm{N}$-out membrane proteins, whereas the number of charges at the $\mathrm{N}$ terminus and the expression level affect the dependency on YidC and PMF.

In the previous report (24), it is concluded that introduction of both negatively and positively charged residues in the $\mathrm{N}$-terminal region of Pf3-Lep renders insertion YidC-dependent. Both net charge numbers and charge distribution are important to determine the YidC-dependence. In this regard, our reconstitution

(C) Effects of PMF on Pf3-Lep (amber) and V15D insertion. $F_{0} F_{1}$-ATPase was coreconstituted with MPIase and YidC to impose PMF. The proteoliposomes thus reconstituted were subjected to Pf3-Lep (amber) and V15D insertion. The insertion activity was determined and is shown at the bottom. In all the autoradiograms, the position of membrane-protected fragment is indicated. Also, the PK-resistant bands unrelated with membrane insertion are indicated by asterisks. 
A

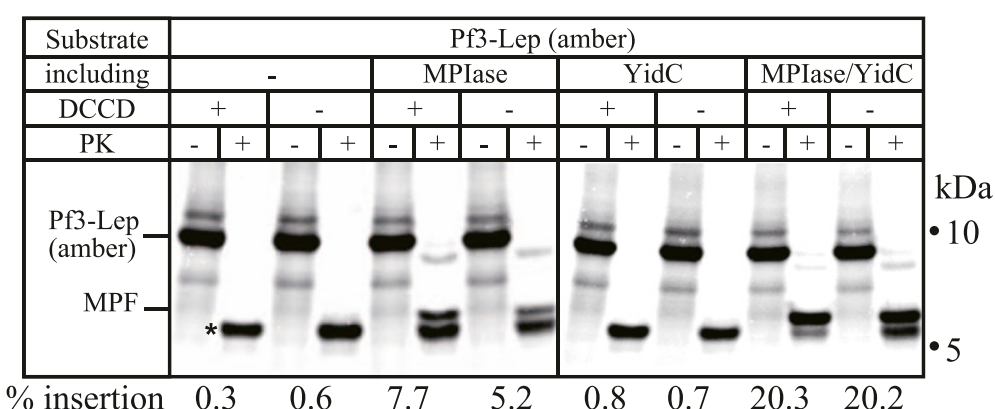
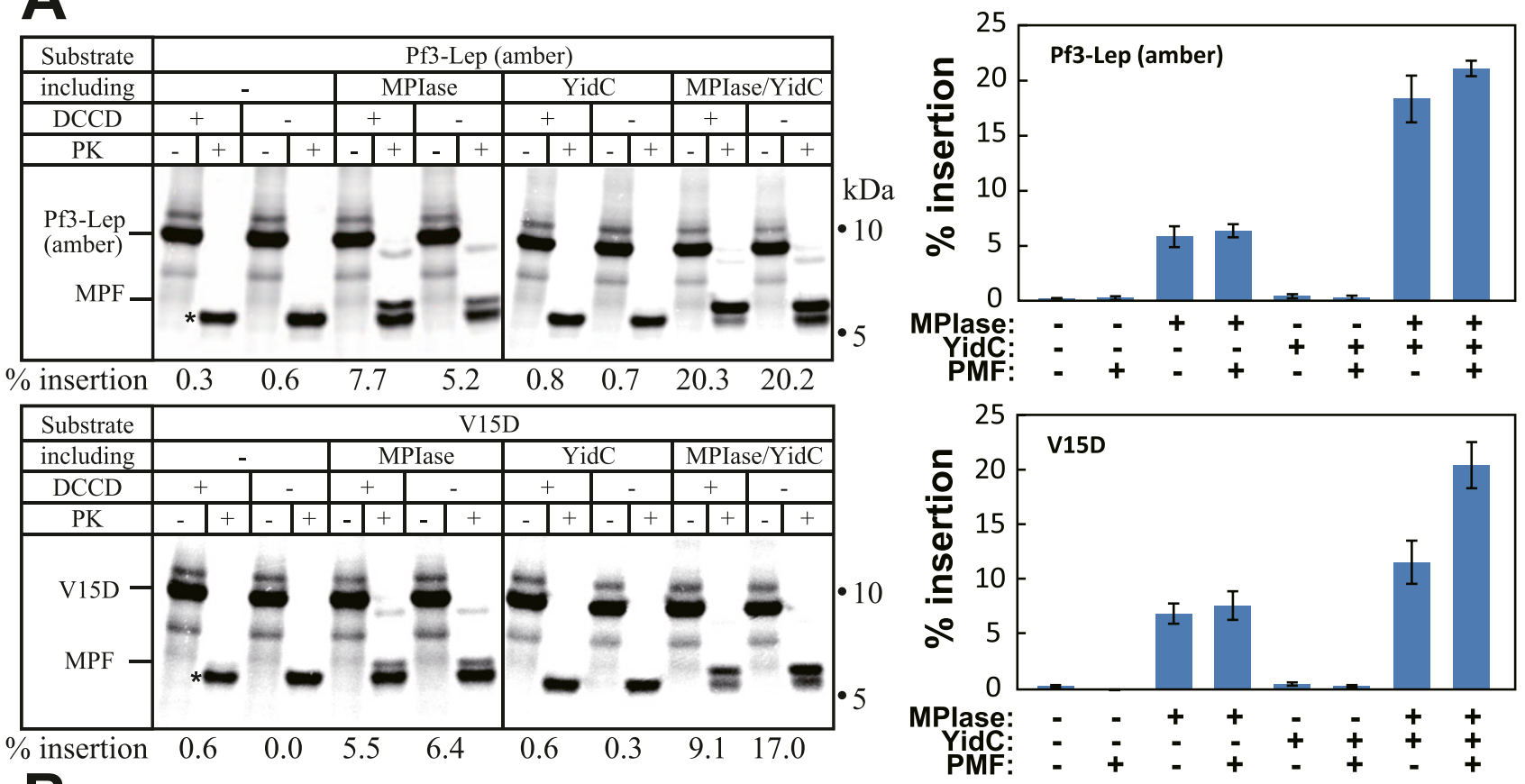

B
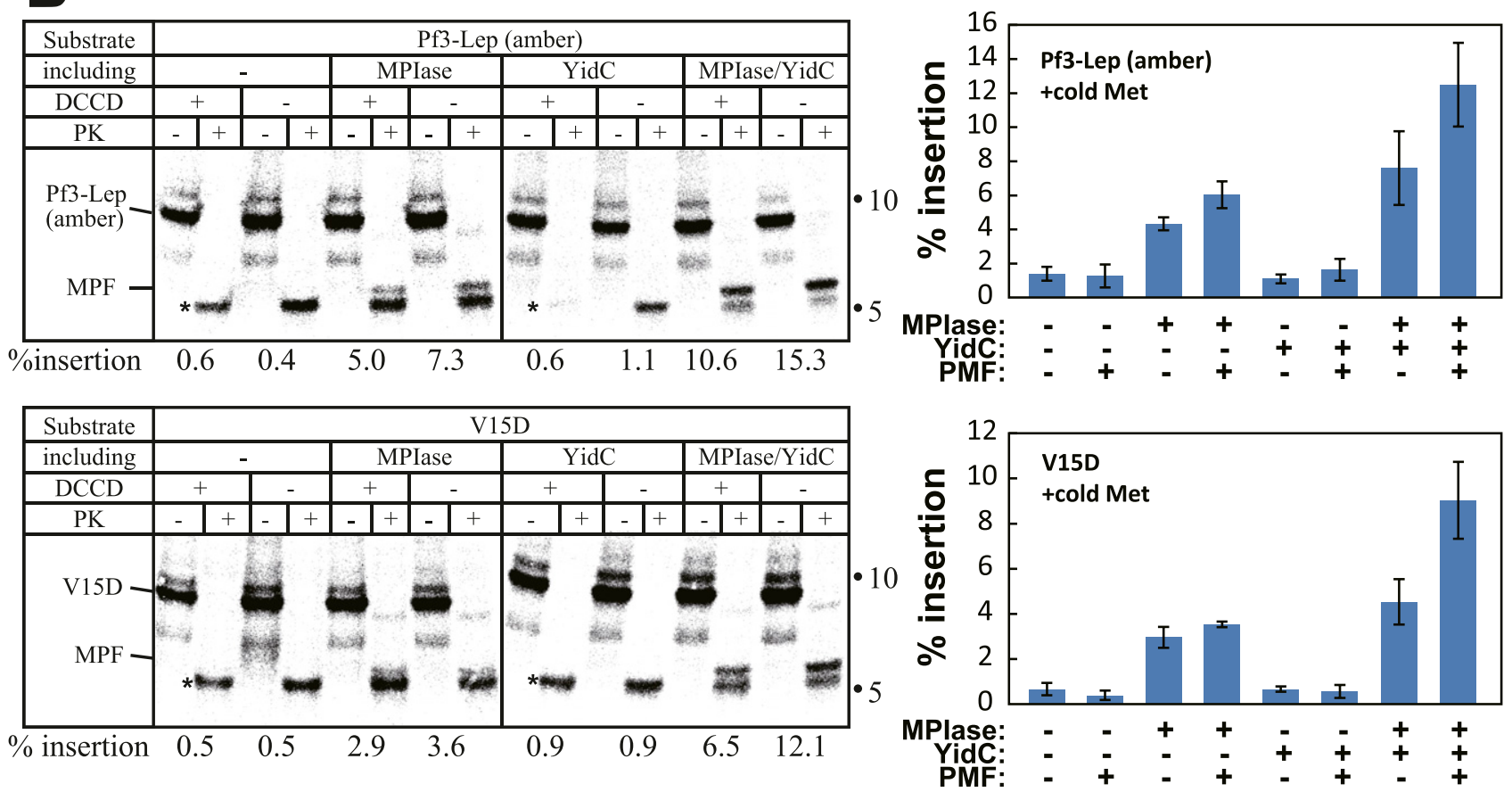

Figure 4. Expression level of the substrates affects the dependency on insertion factors.

(A, B) Pf3-Lep (amber) and V15D insertion into proteoliposomes becomes dependent on YidC and proton motive force as their expression levels increase. (A) The insertion reactions were carried out in the presence of $10 \mathrm{MBq}$ radioactive methionine/ $\mathrm{ml}$ to increase the expression levels of Pf3-Lep (amber) (upper panel) and V15D (lower panel) (A). (B) The expression levels were further increased by adding cold methionine ( $0.3 \mathrm{mM})$ (B). The insertion activity for each lot of proteoliposomes was determined as described in the legend to Fig 1D, shown at the bottom. The position of membrane-protected fragment is indicated. The PK-resistant bands unrelated with membrane insertion are indicated by asterisks. The experiments were carried out at least three times. Average activities with error bars are shown at the right of each autoradiogram.

system would be useful to examine the extent of the dependency precisely. Moreover, it is known that the introduction of positively charged residues, such as V4R and V15R, renders insertion SecYEGdependent in addition (24), strongly suggesting the interplay between
SecYEG and MPlase/YidC/PMF. Again, our reconstitution system would be useful to analyze such an interplay. Analysis of the insertion of additional mutants with charge alterations in the $\mathrm{N}$-terminal region is on progress. 


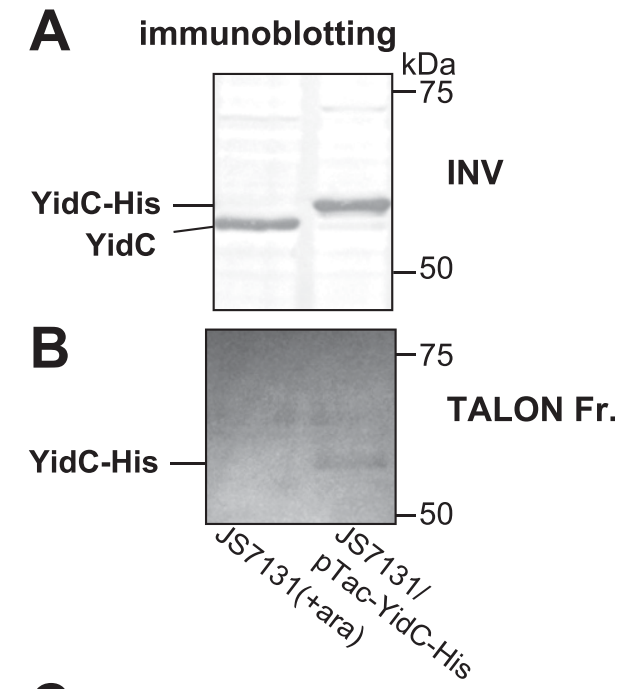

\section{TLC/immunostaining}

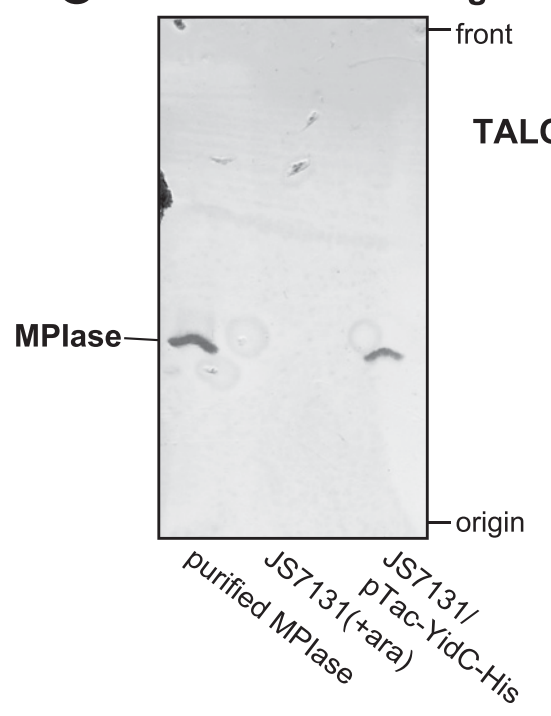

Figure 5. MPlase and YidC interact directly.

(A) Expression level of YidC in INV (10 $\mu$ g protein) prepared from JS7131 and JS7131/pTac-YidC-CHis. It was analyzed by immunoblotting using anti-YidC antibody. (B) Purification of YidC on TALON column chromatography. Solubilized membranes of each INV were applied onto the TALON column, followed by elution with $150 \mathrm{mM}$ imidazole. The eluted fraction $(5 \mu \mathrm{l})$ was subjected to immunoblotting using anti-YidC antibody. (C) Detection of MPlase in the eluted fractions. (B) The eluted fractions in (B) $(5 \mu \mathrm{l})$ were analyzed on TLC, and then visualized by immunostaining using anti-MPlase antibody. Purified MPlase (10 ng) was used as a standard. The positions of TLC origin/front and MPlase are shown.

Source data are available for this figure.

\section{Materials and Methods}

\section{Materials}

INV were prepared from EK413 (29), KS23 (30), and JS7131 (17) cells, as described (31). To deplete MPlase and YidC, conditionally lethal mutants KS23 and JS7131 were cultivated in the absence of arabinose, respectively. Ffh, FtsY, and YidC were purified from overproducer of the respective proteins as described (7). MPlase was purified from MC4100 as described $(15,16) . F_{0} F_{1}$-ATPase from a thermophilic Bacillus PS3 was overproduced in DK8 harboring plasmid pTR19ASDSEAC (32), a kind gift from Dr. Kuruma (JAMSTEC), and then purified on a TALON column, a cobalt metal-affinity column (Clontech), as described (33). The purified preparation of $\mathrm{F}_{0} \mathrm{~F}_{1}$-ATPase was solubilized in $50 \mathrm{mM}$ Hepes/ $\mathrm{KOH}, \mathrm{pH} 7.5,1.5 \% \mathrm{OG}$, and 50\% (wt/vol) glycerol. Plasmids pLZ1-Pf3-Lep after 61 amber codons and pLZ1-Pf3-Lep V15D after 61 amber codons, which carry genes for Pf3-Lep (amber) and V15D (24) (Fig S1) under the control of the $T 7$ promoter, were provided by Prof Dalbey (Ohio State Univ). Plasmids pT7-7-Pf3 (13) and PIVEX-OmpA (34) were used in vitro to synthesize Pf3 coat protein and OmpA, respectively. Plasmid pTac-YidC-CHis (8) was used to express His-tagged YidC. PL (E. coli polar phospholipids) and DAG (dioleoylglycerol) were purchased from Avanti Polar Lipids, Inc. Detergents OG ( $n$-octyl$\beta$-D-glucopyranoside) and DDM ( $n$-dodecyl $\beta$-D-maltoside) were obtained from Dojindo Laboratories. The PURE system, obtained from GeneFrontier Corporation, was optimized for the in vitro inserration assay (15). $\left.{ }^{35} \mathrm{~S}\right]$ EXPRESS Protein Labeling Mix, a mixture containing $\left[{ }^{35} \mathrm{~S}\right]$ methionine and $\left[{ }^{35} \mathrm{~S}\right]$ cysteine $(\sim 37 \mathrm{TBq} / \mathrm{mmol})$, was from Perkin Elmer, Inc.

\section{Reconstitution of (proteo)liposomes}

Proteoliposomes were formed by dialysis as follows. PL (500 $\mu \mathrm{g})$, YidC $\left(40 \mu \mathrm{g}\right.$ ), and $\mathrm{F}_{0} \mathrm{~F}_{1}$-ATPase $(25 \mu \mathrm{g})$, solubilized in $1.5 \%$ (wt/vol) OG, were mixed and incubated on ice for $30 \mathrm{~min}$, followed by dialysis against buffer A (50 mM HEPES-KOH, pH 7.5, 1 mM dithiothreitol) for at least $3 \mathrm{~h}$ at $4^{\circ} \mathrm{C}$. Proteoliposomes, thus reconstituted, were recovered by centrifugation $\left(160,000 \mathrm{~g}, 1 \mathrm{~h}, 4^{\circ} \mathrm{C}\right)$ and suspended in buffer A. DAG-containing liposomes were formed by sonication as described (35). PL and DAG (10\% amount to PL), mixed in the solvent, were dried under a nitrogen stream and then under vacuum. The dried residues were hydrated in buffer $A$ and allowed to form liposomes through sonication. When necessary, MPlase was mixed with PL and DAG to yield MPlase liposomes. Equal amounts of the YidC/ $F_{0} F_{1}$-ATPase proteoliposomes and PL/DAG liposomes or PL/ DAG/MPlase liposomes were mixed, frozen, thawed and then fused through an extruder with filters of $0.4 \mu \mathrm{m}$ pore size, as described $(7,13)$.

\section{Assaying of protein insertion}

The reaction mixture $(20 \mu \mathrm{l})$, containing the PURE system, SRP (50 $\mu \mathrm{g} / \mathrm{ml})$, Fts $(50 \mu \mathrm{g} / \mathrm{ml})$, plasmid DNA, $\left[{ }^{35} \mathrm{~S}\right]$ methionine $(\sim 2 \mathrm{MBq} / \mathrm{ml}$ in Figs 1-3 and $\sim 10 \mathrm{MBq} / \mathrm{ml}$ in Fig 4), and INV or proteoliposomes (0.4 $\mathrm{mg} / \mathrm{ml}$ ), was incubated at $37^{\circ} \mathrm{C}$ for $30 \mathrm{~min}$. Sodium azide $(1 \mathrm{mM})$ or DCCD $(0.15 \mathrm{mM})$ was added when specified. The reaction was terminated by chilling on ice. An aliquot $(3 \mu \mathrm{l})$ was used to monitor the synthesis level. Another aliquot $(15 \mu \mathrm{l})$ was treated with $0.5 \mathrm{mg} / \mathrm{ml}$ proteinase $\mathrm{K}$ (Roche Diagnostics) for $20 \mathrm{~min}$ at $25^{\circ} \mathrm{C}$. The proteins were precipitated with $5 \%$ trichloroacetic acid. After washing the precipitates with acetone, they were analyzed by SDS-PAGE and autoradiography. The radioactive bands were visualized with a Phosphorimager (GE Healthcare) and quantitated using ImageQuant software (GE Healthcare). 


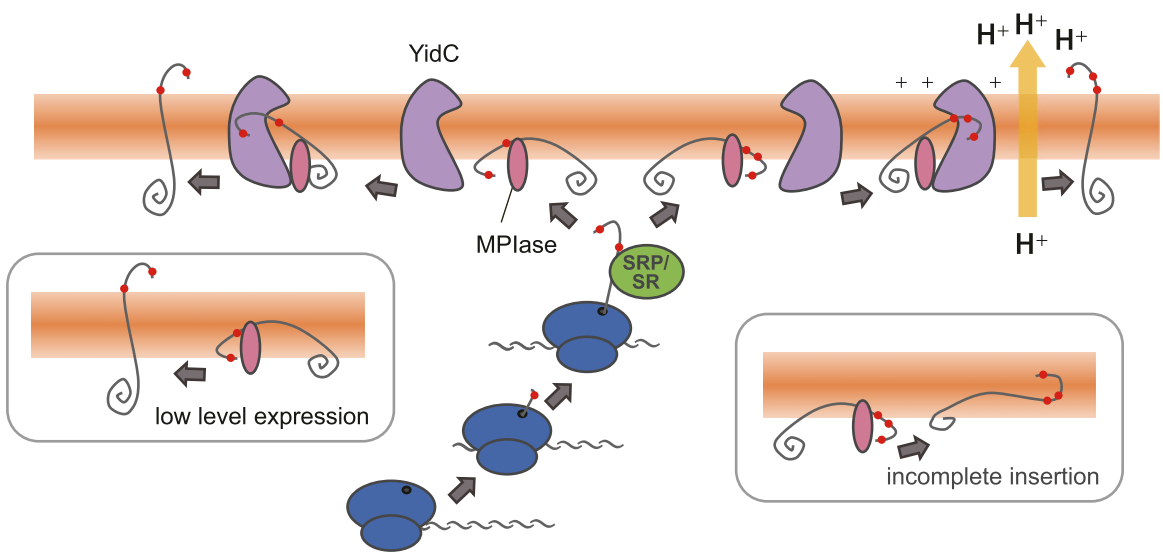

Figure 6. Current model for Pf3-Lep (amber) and V15D insertion into membranes.

When the expression levels of substrates are low, MPlase is sufficient for insertion (left). As the expression levels of the substrates increase, the MPlasedependent insertion being to be stimulated by YidC and proton motive force. The yellow arrow represents the generation of proton motive force. The negatively charged residues in the periplasmic region of Pf3-Lep and V15D are denoted by red dots. See the text for details.

\section{Pull-down assay}

INV were prepared from JS7131 cultivated in the presence of $0.2 \%$ arabinose or J57131/pTac-YidC-CHis in the absence of inducers. Both INV (4 mg protein) were treated with 1\% DDM, 20\% glycerol, $50 \mathrm{mM}$ HEPES-KOH ( $\mathrm{pH} 7.5$ ), followed by recovery of solubilized membranes by centrifugation $\left(170,000 \mathrm{~g}, 30 \mathrm{~min}, 4^{\circ} \mathrm{C}\right)$. They were then applied onto the TALON column $(0.5 \mathrm{ml})$. After washing the columns with $20 \mathrm{ml}$ of $0.02 \%$ DDM, 10\% glycerol, and $50 \mathrm{mM}$ HEPES$\mathrm{KOH}(\mathrm{pH} 7.5)$, bound materials were eluted with the same buffer containing $150 \mathrm{mM}$ imidazole. The YidC and MPlase amounts in the eluted fractions were analyzed by immunoblotting by means of anti-YidC antibody and immunostaining of the TLC plates by means of anti-MPlase antibody, respectively. To detect MPlase, the TLC plates were coated before incubation with antibodies, as described (30).

\section{Supplementary Information}

Supplementary Information is available at https://doi.org/10.26508/lsa. 202101162.

\section{Acknowledgements}

We thank Prof. R. Dalbey, Ohio State University, for the plasmids encoding Pf3-Lep (amber) and the V15D mutant, and for the fruitful discussion; and Ms M Saikudo and Ms M Sawaguchi for the technical assistance. The experiments involving radioisotopes were carried out at the Radio Isotope laboratory of Iwate University. This work was supported by KAKENHI grants (nos. 15KT0073, 17H02209, and 18KK0197 to K Nishiyama, and no. 18J21847 to H Nishikawa). H Nishikawa was a recipient of the Japan Society for the Promotion of Science (JSPS) fellowship.

\section{Author Contributions}

Y Endo: conceptualization, data curation, formal analysis, validation, investigation, visualization, and writing-original draft.

Y Shimizu: investigation and visualization.
H Nishikawa: conceptualization, data curation, formal analysis, funding acquisition, validation, and investigation.

K Sawasato: conceptualization, resources, data curation, and formal analysis.

K Nishiyama: conceptualization, resources, data curation, formal analysis, supervision, funding acquisition, validation, investigation, project administration, and writing-original draft, review, and editing

\section{Conflict of Interest Statement}

The authors declare that they have no conflict of interest.

\section{References}

1. Kiefer D, Kuhn A (2018) YidC-mediated membrane insertion. FEMS Microbiol Lett 365: fny106. doi:10.1093/femsle/fny106

2. Shanmugam SK, Dalbey RE (2019) The conserved role of YidC in membrane protein biogenesis. Microbiol Spectr 7: PSIB-0014-2018. doi:10.1128/microbiolspec.PSIB-0014-2018

3. Denks K, Vogt A, Sachelaru I, Petriman NA, Kudva R, Koch HG (2014) The Sec translocon mediated protein transport in prokaryotes and eukaryotes. Mol Membr Biol 31: 58-84. doi:10.3109/09687688.2014.907455

4. Endo Y, Nishiyama K (2015) Relationship between glycolipozyme MPlase and components comprising the protein transport machinery. Med Res Arch 2: 11. https://esmed.org/MRA/mra/article/view/403

5. Nishiyama K, Shimamoto K (2014) Glycolipozyme membrane protein integrase (MPlase): Recent data. Biomol Concepts 5: 429-438. doi:10.1515/bmc-2014-0030

6. Nishiyama K, Ikegami A, Moser M, Schiltz E, Tokuda H, Müller M (2006) A derivative of lipid A is involved in signal recognition particle/SecYEGdependent and -independent membrane integrations. J Biol Chem 281: 35667-35676. doi:10.1074/jbc.M608228200

7. Sasaki M, Nishikawa H, Suzuki S, Moser M, Huber M, Sawasato K, Matsubayashi HT, Kumazaki K, Tsukazaki T, Kuruma Y, et al (2019) The bacterial protein YidC accelerates MPlase-dependent integration of membrane proteins. J Biol Chem 294: 18898-18908. doi:10.1074] jbc.RA119.011248

8. Nishikawa H, Sasaki M, Nishiyama KI (2017) Membrane insertion of FO C subunit of FOF1 ATPase depends on glycolipozyme MPlase and is 
stimulated by YidC. Biochem Biophys Res Commun 487: 477-482. doi:10.1016/j.bbrc.2017.04.095

9. Nishikawa H, Kanno K, Endo Y, Nishiyama KI (2021) Ring assembly of c subunits of F0 F1 -ATP synthase in Propionigenium modestum requires YidC and Uncl following MPlase-dependent membrane insertion. FEBS Lett 595: 647-654. doi:10.1002/1873-3468.14036

10. Kiefer D, Kuhn A (1999) Hydrophobic forces drive spontaneous membrane insertion of the bacteriophage Pf3 coat protein without topological control. EMBO J 18: 6299-6306. doi:10.1093/emboj/18.22.6299

11. Robinson PJ, Woolhead CA (2013) Post-translational membrane insertion of an endogenous YidC substrate. Biochim Biophys Acta 1833: 2781-2788. doi:10.1016/j.bbamcr.2013.07.003

12. Geller BL, Wickner W (1985) M13 procoat inserts into liposomes in the absence of other membrane proteins. J Biol Chem 260: 13281-13285. doi:10.1016/s0021-9258(17)38867-1

13. Kawashima Y, Miyazaki E, Müller M, Tokuda H, Nishiyama K (2008) Diacylglycerol specifically blocks spontaneous integration of membrane proteins and allows detection of a factor-assisted integration. J Biol Chem 283: 24489-24496. doi:10.1074/jbc.M801812200

14. Stiegler N, Dalbey RE, Kuhn A (2011) M13 procoat protein insertion into YidC and SecYEG proteoliposomes and liposomes. J Mol Biol 406: 362-370. doi:10.1016/j.jmb.2010.12.036

15. Nishiyama K, Maeda M, Abe M, Kanamori T, Shimamoto K, Kusumoto S, Ueda T, Tokuda H (2010) A novel complete reconstitution system for membrane integration of the simplest membrane protein. Biochem Biophys Res Commun 394: 733-736. doi:10.1016/j.bbrc.2010.03.061

16. Nishiyama K, Maeda M, Yanagisawa K, Nagase R, Komura H, Iwashita T, Yamagaki T, Kusumoto S, Tokuda H, Shimamoto K (2012) MPlase is a glycolipozyme essential for membrane protein integration. Nat Commun 3: 1260. doi:10.1038/ncomms2267

17. Samuelson JC, Chen M, Jiang F, Möller I, Wiedmann M, Kuhn A, Phillips GJ, Dalbey RE (2000) YidC mediates membrane protein insertion in bacteria. Nature 406: 637-641. doi:10.1038/35020586

18. Kumazaki K, Chiba S, Takemoto M, Furukawa A, Nishiyama K, Sugano Y, Mori T, Dohmae N, Hirata K, Nakada-Nakura Y, et al (2014) Structural basis of Sec-independent membrane protein insertion by YidC. Nature 509: 516-520. doi:10.1038/nature13167

19. Chen M, Samuelson JC, Jiang F, Muller M, Kuhn A, Dalbey RE (2002) Direct interaction of YidC with the Sec-independent Pf3 coat protein during its membrane protein insertion. J Biol Chem 277: 7670-7675. doi:10.1074/ jbc.M110644200

20. Kiefer D, Hu X, Dalbey R, Kuhn A (1997) Negatively charged amino acid residues play an active role in orienting the Sec-independent Pf3 coat protein in the Escherichia coli inner membrane. EMBO J 16: 2197-2204. doi:10.1093/emboj/16.9.2197

21. Samuelson JC, Jiang F, Yi L, Chen M, de Gier JW, Kuhn A, Dalbey RE (2001) Function of YidC for the insertion of M13 procoat protein in Escherichia coli: Translocation of mutants that show differences in their membrane potential dependence and Sec requirement. J Biol Chem 276: 34847-34852. doi:10.1074/jbc.M105793200

22. Serek J, Bauer-Manz G, Struhalla G, van den Berg L, Kiefer D, Dalbey R, Kuhn A (2004) Escherichia coli YidC is a membrane insertase for Secindependent proteins. EMBO J 23: 294-301. doi:10.1038/sj.emboj.7600063
23. van der Laan M, Bechtluft P, Kol S, Nouwen N, Driessen AJ (2004) F1F0 ATP synthase subunit $c$ is a substrate of the novel YidC pathway for membrane protein biogenesis. J Cell Biol 165: 213-222. doi:10.1083/ jcb.200402100

24. Zhu L, Wasey A, White SH, Dalbey RE (2013) Charge composition features of model single-span membrane proteins that determine selection of YidC and SecYEG translocase pathways in Escherichia coli. I Biol Chem 288: 7704-7716. doi:10.1074/jbc.M112.429431

25. Koch HG, Hengelage T, Neumann-Haefelin C, MacFarlane J, Hoffschulte HK, Schimz KL, Mechler B, Müller M (1999) In vitro studies with purified components reveal signal recognition particle (SRP) and SecA/SecB as constituents of two independent protein-targeting pathways of Escherichia coli. Mol Biol Cell 10: 2163-2173. doi:10.1091/mbc.10.7.2163

26. Oliver DB, Cabelli RJ, Dolan KM, Jarosik GP (1990) Azide-resistant mutants of Escherichia coli alter the SecA protein, an azide-sensitive component of the protein export machinery. Proc Natl Acad Sci U S A 87: 8227-8231. doi:10.1073/pnas.87.21.8227

27. Hermolin J, Fillingame RH (1989) H+-ATPase activity of Escherichia coli F1F0 is blocked after reaction of dicyclohexylcarbodiimide with a single proteolipid (subunit c) of the F0 complex. J Biol Chem 264: 3896-3903. doi:10.1016/s0021-9258(19)84937-2

28. Kumazaki K, Kishimoto T, Furukawa A, Mori H, Tanaka Y, Dohmae N, Ishitani R, Tsukazaki T, Nureki O (2014) Crystal structure of Escherichia coli YidC, a membrane protein chaperone and insertase. Sci Rep 4: 7299. doi:10.1038/srep07299

29. Nishiyama K, Suzuki T, Tokuda H (1996) Inversion of the membrane topology of SecG coupled with SecA-dependent preprotein translocation. Cell 85: 71-81. doi:10.1016/s0092-8674(00)81083-1

30. Sawasato K, Sato R, Nishikawa H, limura N, Kamemoto Y, Fujikawa K, Yamaguchi T, Kuruma Y, Tamura Y, Endo T, et al (2019) CdsA is involved in biosynthesis of glycolipid MPlase essential for membrane protein integration in vivo. Sci Rep 9: 1372. doi:10.1038/s41598-018-37809-8

31. Alami M, Trescher D, Wu LF, Müller M (2002) Separate analysis of twinarginine translocation (Tat)-specific membrane binding and translocation in Escherichia coli. J Biol Chem 277: 20499-20503. doi:10.1074/jbc.M201711200

32. Kuruma Y, Suzuki T, Ono S, Yoshida M, Ueda T (2012) Functional analysis of membranous Fo-a subunit of F1Fo-ATP synthase by in vitro protein synthesis. Biochem J 442: 631-638. doi:10.1042/BJ20111284

33. Berhanu S, Ueda T, Kuruma Y (2019) Artificial photosynthetic cell producing energy for protein synthesis. Nat Commun 10: 1325. doi:10.1038/s41467-019-09147-4

34. Nishiyama K, Tokuda H (2010) Preparation of a highly translocationcompetent proOmpA/SecB complex. Protein Sci 19: 2402-2408. doi:10.1002/pro.520

35. Nishikawa H, Sasaki M, Nishiyama KI (2020) In vitro assay for bacterial membrane protein integration into proteoliposomes. Bio Protoc 10 : e3626. doi:10.21769/BioProtoc.3626

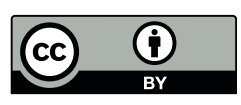

License: This article is available under a Creative Commons License (Attribution 4.0 International, as described at https://creativecommons.org/ licenses/by/4.0/). 